Usages du français et pratiques d'enseignement en Europe balkanique, centrale et orientale - Grèce,

Serbie, Bulgarie, Moldavie, Hongrie, Allemagne, Russie - XVIIIe - XXe siècles

\title{
La présence éducative et culturelle de la congrégation de Saint Joseph de l'Apparition en Grèce. Le cas de l'école Jeanne d'Arc du Pirée
}

Aikaterini Lalagianni et Vassiliki Lalagianni

\section{(2) OpenEdition}

Édition électronique

URL : https://journals.openedition.org/dhfles/4173

DOI : $10.4000 /$ dhfles. 4173

ISSN : 2221-4038

Éditeur

Société Internationale pour l'Histoire du Français Langue Étrangère ou Seconde

Édition imprimée

Date de publication : 1 juin 2015

Pagination : 79-96

ISSN : 0992-7654

Référence électronique

Aikaterini Lalagianni et Vassiliki Lalagianni, « La présence éducative et culturelle de la congrégation de Saint Joseph de l'Apparition en Grèce. Le cas de l'école Jeanne d'Arc du Pirée », Documents pour I'histoire du français langue étrangère ou seconde [En ligne], 54 | 2015, mis en ligne le 01 janvier 2018 , consulté le 27 mars 2023. URL : http://journals.openedition.org/dhfles/4173 ; DOI : https://doi.org/ $10.4000 /$ dhfles. 4173

Ce document a été généré automatiquement le 27 mars 2023.

Tous droits réservés 


\title{
La présence éducative et culturelle de la congrégation de Saint Joseph de l'Apparition en Grèce. Le cas de l'école Jeanne d'Arc du Pirée
}

\author{
Aikaterini Lalagianni et Vassiliki Lalagianni
}

1 Les écoles congréganistes en Grèce, fondées, pour la plupart, durant l'époque ottomane dans diverses régions du pays, ont une longue histoire. La raison d'être de ces écoles trouve son origine dans une tradition éducative celle des sœurs et des frères chrétiens, catholiques ou protestants, qui ont adopté la politique de création d'écoles en l'adaptant aux besoins d'une éducation destinée aux filles ou aux garçons de chaque

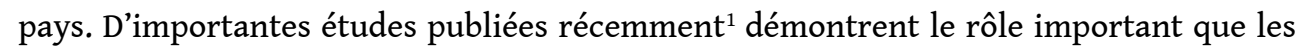
écoles fondées par les ordres catholiques français ont joué dans le bassin méditerranéen.

\section{La création des écoles congréganistes françaises en Grèce}

2 L'étude des écoles catholiques fondées par des frères ou des sœurs français est un sujet complexe car il touche une série d'autres sujets concernant la fondation de l'état hellénique au XIX ${ }^{\mathrm{e}}$ siècle, la création de l'identité nationale du nouvel État ainsi que le rôle que joua l'Église orthodoxe grecque. Il est sujet complexe car lié aux politiques éducatives du nouvel État, à l'attitude de celui-ci envers ces écoles et aux conséquences de la présence d'une communauté catholique au sein d'une société relativement homogène du point de vue religieux.

3 Sous l'occupation ottomane, les premières écoles congréganistes sont fondées d'abord dans les îles de la mer Égée et surtout dans les Cyclades. Il y avait en Grèce de grandes communautés catholiques depuis des siècles : Francs et Vénitiens ont, après la défaite de Byzance en 1204, occupé des territoires de l'Empire et ont créé des états et des 
principautés. Beaucoup de communautés catholiques nées à cette époque, sont demeurées en activité durant les siècles, surtout dans les îles de la mer Égée et dans les îles ioniennes. Dans ces îles, les congrégations ont trouvé un terrain propice pour la création des écoles catholiques. En ce qui concerne les écoles congréganistes fondées par des religieuses, les premières venues étaient les Ursulines qui s'installent en 1739 sur l'île de Naxos, où la présence des Francs et plus tard des Vénitiens avait favorisé l'essor d'une communauté catholique. Elles seront suivies par les Lazaristes, implantés eux aussi à Naxos mais aussi à Santorin (Théra) et à Salonique en 1783. Les écoles catholiques se multiplient au XIX ${ }^{\mathrm{e}}$ siècle, et surtout dans sa deuxième moitié, atteignant aussi la Grèce continentale : ainsi les Filles de la Charité s'installent à Santorin (1841), les Sœurs de Saint Joseph de l'Apparition à Syros (Syra) en 1846, les Sœurs de NotreDame de la Compassion à Céphalonie (1857), les Sœurs du Bon Pasteur à Patras (1858), les Frères des Écoles chrétiennes (Lassaliens) à Syros (1858), les Sœurs du Sacré-Cœur à Rhodes (1875), les Salésiens à Athènes (1891), les Sœurs de Saint Joseph de Lyon à Samos, (1901), les Frères Maristes à Mytilène (1901) et la Société des Missions africaines de Lyon à Samos en 1891 (Antoniou 2011 : 34-36). Les écoles dirigées par des sœurs et frères français catholiques se développent au sein d'une société où l'Église orthodoxe joue un rôle primordial dans la création de la conscience identitaire néo-hellénique. Au $\mathrm{XIX}^{\mathrm{e}}$ siècle, l'émergence des nationalismes balkaniques, l'indépendance des nations de la région après leur libération des Ottomans et la création des états-nations, exigeaient un système éducatif qui renforcerait l'identité nationale hellénique au sein d'une Europe qui entendait jouer un rôle politique dans le nouvel État grec. De l'autre côté, il existait en Grèce un mouvement propice à

l' "occidentalisation" de la société hellénique, renforcé par l'intelligentsia de la Diaspora, celle-ci étant sous l'influence des idées libérales du siècle des Lumières. Ce nationalisme grec éclairé ayant adopté une vue eurocentrique, prônait "l'européanisation du langage politique » des milieux intellectuels et littéraires mais aussi une européanisation des structures sociales, de l'éducation et de la vie quotidienne de la nation (Myrogiannis $2012: 131)^{2}$. La langue et la culture françaises, liées aux idées humanistes et libérales, était devenue la langue des intellectuels et des élites grecques (Provata 2011: 281-292). Dans le contexte politique et social dichotomique du XIX ${ }^{e}$ siècle, l'installation des écoles catholiques se fit assez facilement en Grèce continentale et insulaire, au moins au début de leur arrivée dans la région. L'autonomisation de l'église orthodoxe grecque du Patriarcat de Constantinople en 1850 marque le passage d'une orthodoxie œcuménique à une orthodoxie nationale (Kavallierakis $2008: 221$ ) et l'Église en Grèce « se nationalise » de plus en plus, devenant un élément décisif dans la formation du nouvel état grec. Aussi les écoles catholiques rencontrent quelquefois, sous la pression de l'église orthodoxe grecque, l'hostilité des rouages étatiques concernant la politique éducative car pour l'État, protéger l'Église signifiait protéger un axe fondamental de l'identité nationale ${ }^{3}$.

4 Les écoles des congrégations religieuses utilisant des méthodes d'enseignement et des curricula différents des écoles grecques et offrant leur enseignement en français, ont provoqué la méfiance de l'État. Dès la fin du XIX ${ }^{e}$ siècle jusqu'à la Seconde Guerre mondiale, une série de lois et de décrets a essayé soit d'encadrer les écoles religieuses dans le système hellénique, soit de contrôler leur fonctionnement - attitude qui montre bien la méfiance de l'État et de l'Église envers ces écoles ${ }^{4}$. L'introduction dans l'enseignement du catéchisme a provoqué, on l'a dit, des réactions non seulement de la part des écoles catholiques mais aussi de la part des ambassades étrangères en Grèce. 
Ces tensions ont duré jusqu'au début du XXe siècle. La loi 4862 /1931 «Sur les écoles étrangères " montre bien un durcissement de la position grecque envers les écoles catholiques, puisqu'elle prévoit l'enseignement obligatoire du grec et du dogme orthodoxe par des professeurs orthodoxes de nationalité hellénique. Dans l'aprèsguerre, on assiste à une réorganisation des écoles en Grèce afin de régler les problèmes qui s'étaient accumulés; il existe aussi une tendance à améliorer les relations avec les pays de l'Europe occidentale, alliés de la Grèce contre le nazisme. Un grand nombre d'accords culturels bilatéraux a favorisé l'implantation française en Grèce tant dans le domaine de la recherche (Institut Pasteur, diverses bourses du gouvernement français, hôpital français) que dans le domaine de l'éducation (Flitouris 2005 : 173-174). Après la fin de la dictature en Grèce (1967-1974), une période de fonctionnement régularisé s'installe, les lois devenant plus tolérantes et la société grecque plus ouverte au pluriculturalisme (Antoniou 2011: 101). Une série de lois et d'ordonnances ${ }^{5}$ sur les écoles étrangères en Grèce, développées surtout après l'entrée de la Grèce dans la Communauté Européenne en 1979, ont réexaminé le cadre institutionnel qui régit le statut de ces écoles et ont contribué à améliorer le fonctionnement des écoles congréganistes françaises, celles-ci faisant un effort pour ne pas afficher leur caractère religieux tout en focalisant sur l'éducation et sur les actes de charité.

\section{L'école Jeanne d'Arc, une école pluriculturelle}

5 La congrégation missionnaire de Saint Joseph de l'Apparition, fondée en France en 1832 par sainte Émilie de Vialar, s'installe en Grèce en 1846. Elle crée une école primaire pour filles à Syros, en 1846 et un établissement secondaire pour filles en 1848 à Chios. Les Sœurs de Saint Joseph de l'Apparition ont fondé par la suite un grand nombre d'écoles partout en Grèce mais surtout dans les îles, où la présence des minorités catholiques demeurait toujours importante ${ }^{6}$. Ces écoles ont connu et connaissent encore une grande prospérité: Syros (1846-1920), Chios (1848-1981), La Canée (1852-1981), Le Pirée (1859 jusqu'à aujourd'hui), Athènes (1856 jusqu'à aujourd'hui), Héraklion (1902-1940), Volos (1904 jusqu'à aujourd'hui), Laurion (1909-1924). Les écoles de la congrégation continuent toujours leur œuvre éducative à Athènes et au Pirée.

6 La préférence de la congrégation à organiser des missions dans les régions ottomanes ne marque pas tant sa volonté de faire du prosélytisme que celle de soutenir l'enseignement et l'instruction religieuse des enfants des communautés catholiques locales $^{7}$ - les sœurs étant venues dans les régions où existait la population catholique. Le plus souvent, elles étaient invitées par une institution catholique sur place ou par l'église catholique comme c'est le cas d'Athènes; au Pirée, c'était l'amiral de la flotte française, qui occupa le port pendant la guerre d'Orient de 1854 à 1857, qui avait appelé les religieuses afin qu'elles instruisent les enfants des Français du Pirée (Kavallierakis $2008: 168)$.

7 Les archives de l'école Jeanne d'Arc du Pirée nous fournissent des informations sur l'éducation des filles dans l'établissement : informations sur l'organisation, les matières enseignées, le règlement et l'orientation de l'enseignement proposé par l'école. Deux notices sur l'histoire de l'école tenues par les supérieures et intitulées Historiques I et Historiques II, nous fournissent aussi des renseignements importants sur l'école depuis sa création, en 1859 , jusqu'en $1975^{\circ}$. 
Dans ces cahiers, les supérieures notaient tout événement important pour l'école, et quelquefois des informations sur les sœurs, sur les invités de l'école ainsi que sur les enseignants. Ces cahiers renvoient aussi à des documents dans lesquels sont consignés de façon parfois allusive mais souvent détaillée, les différents projets et activités de la communauté, les événements qui touchent de près l'école. Les premières années étaient très difficiles pour l'école du Pirée, qui ne disposait pas de ressources financières. La directrice de l'école d'Athènes, sœur Jeanne Blancal, fit d'énormes efforts afin de financer le développement de l'école du Pirée. D'après les Archives de l'école, même la reine olga de Grèce, sollicitée par sœur Blancal, avait soutenu financièrement l'école (Historiques I : 8-9).

Le Pirée n'était à cette époque qu'une ville modeste. Après qu'Athènes fut devenue la capitale du nouvel État grec, le port a connu un essor important. En 1888, il y avait presque 40000 habitants; des bureaux d'assurance et des chambres de commerce s'y sont installés et un grand nombre de sociétés, françaises, anglaises, égyptiennes et italiennes y avaient leur siège. De nombreuses manufactures et petites entreprises ont commencé à s'implanter dans les alentours de la ville (Cambouroglou 1883 : 38-47). Bien que la composition sociale du Pirée soit différente de celle d'Athènes, une classe bourgeoise commence à s'y former, ayant envie de faire instruire ses enfants. C'est dans ce cadre social que se développe l'école des sœurs du Pirée. En 1898, l'école comprenait 200 élèves, 15 élèves en pensionnat et 20 orphelines dans l'orphelinat qui fonctionnait parallèlement, et un grand nombre d'élèves externes (Lacroix 1898: 27). Nous constatons donc que les filles n'appartenaient pas seulement à des familles riches, à une élite locale mais aussi à des couches sociales moins aisées. Le plus grand nombre des élèves provenaient des familles catholiques installées au Pirée pour travailler ; la flotte française qui pendant la guerre d'Orient occupait le port de 1854 à 1857, fournissait aussi des élèves à l'école. On y trouvait également un grand nombre de filles de familles orthodoxes de la moyenne bourgeoisie qui désiraient une éducation « occidentale » à la française pour leurs enfants. L'école était tellement sollicitée que la supérieure notait : «[...] j'ai pris des laïques pour nous aider dans les classes ... » (Historiques $I: 180)$.

Dans les Historiques et surtout dans les Archives de l'école, on trouve de nombreux renseignements concernant la confession et la nationalité des élèves, des statistiques qui confirment l'aspect multiculturel de l'école du Pirée. Il est clair que les sœurs proposent une éducation et un enseignement chrétiens sans prosélytisme, ouvert à des élèves issus de diverses communautés religieuses.

\begin{tabular}{|l|l|l|l|l|l|}
\hline Confession & $1922 / 1923$ & $1923 / 1924$ & $1924 / 1925$ & $1925 / 1926$ & Total \\
\hline Orthodoxes & 478 & 481 & 281 & 316 & 1556 \\
\hline Catholiques & 200 & 95 & 110 & 108 & 513 \\
\hline Grégoriennes & 22 & & 13 & 19 & 54 \\
\hline Israélites & & 2 & 3 & 3 & 8 \\
\hline Protestantes & & & 2 & 2 & 4 \\
\hline
\end{tabular}




\begin{tabular}{|l|l|l|l|l|l|}
\hline Ottomanes & & & & 2 & 2 \\
\hline Autres & 12 & & 24 & 19 & 55 \\
\hline Total & 712 & 518 & 433 & 469 & 2192 \\
\hline
\end{tabular}

La composition de l'école Saint Joseph du Pirée selon l'appartenance religieuse (1922-1926) (Lianou $2013: 512)$

\begin{tabular}{|c|c|c|c|c|c|}
\hline Nationalité & $1922 / 1923$ & $1923 / 1924$ & $1924 / 1925$ & $1925 / 1926$ & Total \\
\hline Grecques & 613 & 450 & 314 & 355 & 1712 \\
\hline Françaises & 10 & 5 & 18 & 17 & 50 \\
\hline Italiennes & 32 & 12 & 17 & 14 & 75 \\
\hline Armeniennes & 48 & 104 & 30 & 38 & 220 \\
\hline Russes & 8 & 7 & 6 & 3 & 24 \\
\hline Albanaises & & & 2 & 2 & 4 \\
\hline Portuguaises & & & 1 & & 1 \\
\hline Ottomanes & & & 3 & 9 & 12 \\
\hline Anglaises & & & 11 & 8 & 19 \\
\hline Serbes & & & & 1 & 1 \\
\hline Autrichiennes & & & & 1 & 1 \\
\hline Bulgares & & & & 2 & 2 \\
\hline Autres & & & 31 & 38 & 69 \\
\hline Total & 712 & 578 & 433 & 468 & 2191 \\
\hline
\end{tabular}

La composition de l'école Saint Joseph du Pirée selon la nationalité, 1922-1926 (Lianou 2013 : 512-513)

11 L'augmentation des Grégoriennes (Arméniennes) à l'école pendant les années 1920-1926 est due aux persécutions du peuple arménien en Turquie qui a conduit à leur expatriation et à leur migration vers la Grèce et d'autres pays européens. Il faut aussi mentionner les bonnes relations de l'école avec les communautés juives du Pirée et d'Athènes. En l'absence d'école de l'Alliance israélite à Athènes, les juifs scolarisaient leurs filles chez les sœurs du Pirée ${ }^{9}$. 


\section{Une éducation pour les filles}

12 La création des écoles par les sœurs catholiques dans le bassin méditerranéen ottoman était à leurs yeux d'une part l'occasion d'offrir une éducation chrétienne au sein de l'Empire ottoman et d'autre part, un moyen pour aider les pays pauvres ${ }^{10}$. En Grèce, les écoles des sœurs ont joué un rôle important dans la scolarisation des filles au XIX ${ }^{\mathrm{e}}$ et au début du XX siècle.

13 La loi de 1834 sur les écoles primaires a rendu l'enseignement primaire obligatoire sans aucune distinction entre les deux sexes ${ }^{11}$. Cependant, malgré cette loi qui prévoyait la scolarisation des deux sexes et malgré l'expansion rapide des écoles primaires, le pourcentage de la participation des filles dans l'enseignement primaire reste faible, durant le XIX ${ }^{\mathrm{e}}$ siècle.

14 En ce qui concerne l'enseignement secondaire, seule la scolarisation des garçons était prévue par le décret de 1836 qui en excluait les filles ${ }^{12}$. Dans le nouvel état hellénique et tout au long du XIX ${ }^{\mathrm{e}}$ siècle, il existe une philosophie et une stratégie différentes quant à l'enseignement féminin, qui avait comme obligation principale non pas l'éducation de futures citoyennes mais la préparation de mères parfaites et de jeunes filles sages capables de diriger la maison et de gérer la vie quotidienne. L'état n'accordait pas une grande importance à l'éducation des filles au niveau secondaire ; ainsi, l'enseignement des filles est devenu l'affaire des écoles étrangères établies en Grèce, c'est-à-dire les écoles privées destinées aux filles (П $\rho \theta \varepsilon v \alpha \gamma \omega \gamma \varepsilon i ́ \alpha)$ ou les écoles des congrégations (Ziogou-Karastergiou 1986 : 65). L'Église grecque, malgré ses réactions contre les écoles catholiques et protestantes, n'a pas créé d'écoles destinées à l'enseignement féminin, se contentant des heures de catéchisme dispensées par les prêtres dans les églises ou dans les locaux appartenant à l'église, ou par les professeurs du secondaire qui enseignaient la théologie dans les écoles. Elle regardait avec méfiance l'éducation des filles, et la considérait comme l'un des symptômes de la «corruption » due à l'occidentalisation des mœurs dans la société hellénique (Kavallierakis $2008: 221$ ).

Vers la fin du XIXe siècle, des écoles d'état ont été créées pour la formation des filles. Ces écoles appelées "didaskaleia " s'adressaient à des filles issues de couches sociales moyennes ou pauvres. La profession d'institutrice fut très recherchée par les filles durant le XIX ${ }^{\text {e }}$ siècle, car elle leur permettait de gagner leur vie avec dignité mais aussi de sortir dans la sphère publique. Les écoles des sœurs étaient très sollicitées par les filles car elles étaient renommées pour leur enseignement; en plus, l'apprentissage du français à cette époque était considéré comme un grand avantage pour obtenir un travail. Les écoles congréganistes bien qu'elles «s'adressaient initialement à la seule population catholique des villes où elles se sont installées, elles se sont progressivement ouvertes pour accueillir dans leurs rangs [...] les filles des élites urbaines locales, emportées par le climat de francisation qui s'était emparé de la société grecque et séduites par le programme d'études dispensé en langue française » (Provata $2012: 180)$.

16 D’après Filippoussis \& Pappas, le fait que les écoles des sœurs utilisaient dans leurs manuels scolaires la langue parlée, dite « démotique » et non pas la langue savante, dite " katharevousa ", fut aussi une des raisons de l'attrait qu'exerçaient ces établissements à la population féminine (Filippoussis \& Pappas $2010: 50$ ). 

enseignement, une forme d'enseignement secondaire pour les filles, dans la Grèce du $\mathrm{XIX}^{\mathrm{e}}$ siècle $^{13}$. En ce domaine, les écoles des Sœurs de Saint Joseph ont joué un rôle primordial dans la charte éducative tout au long du XIXe siècle. Leur apport à l'enseignement des filles est crucial et constitue une contribution ultime à la création d'un climat positif dans la société hellénique pour la scolarisation des filles après l'école primaire. Un facteur qui a contribué de manière décisive à l'intégration de l'école de Saint Joseph du Pirée et en général de toutes les écoles de la congrégation dans les sociétés locales, était l'enseignement à orientation professionnelle que ces écoles offraient aux filles grecques.

En 1900, la supérieure de l'école Célina Joseph Le Bouffo, écrit dans les carnets :

Les cours suivis par nos élèves sont aussi complets que possible. La langue française en est la base. Chaque année on présente des élèves pour des brevets de capacité à la Commission de l'École française d'Athènes [...]. La plupart de ces jeunes filles brevetées se placent comme institutrices dans des familles, dans les écoles grecques, jusque dans les provinces et les îles les plus reculées de la Grèce. (Historiques I : 3-4)

Dans ce passage nous constatons que déjà en 1900 la scolarisation dans l'école des sœurs était liée à une orientation professionnelle dont les sœurs étaient fières. Les références aux examens de l'École française d'Athènes, qui sont fréquentes dans les Historiques, soulignent la volonté des sœurs de montrer d'une façon officielle que leur école et leurs activités éducatives se trouvaient sous l'égide française.

\section{«Former, soigner, assister »14}

La fondatrice de l'ordre Émilie de Vialar souligne le besoin de se consacrer aux œuvres de charité : «instruire [...] les enfants et surtout les enfants pauvres; [et] soigner les malades à domicile, dans les hôpitaux, dans les prisons ... » (Langlois 1984 : 441).

Les activités de charité caractérisent toute l'histoire de l'école du Pirée, en même temps que les activités éducatives et culturelles. La politique de scolariser gratuitement des enfants pauvres, d'accueillir et de soigner les orphelins, un état d'esprit d'activité philanthropique et de dévouement pour adoucir les douleurs de l'Autre, ont eu comme résultat de rendre les sœurs très aimées des sociétés dans lesquelles elles œuvraient. Pendant les périodes difficiles pour le peuple grec, les écoles des Sœurs de Saint Joseph se sont trouvées à ses côtés. Les Historiques nous informent que durant les périodes de guerre, la plupart des familles ne pouvaient pas payer les frais de scolarisation de leurs enfants : les sœurs acceptaient tous les enfants sans distinction et faisaient des efforts pour maintenir l'école en fonctionnement. Pendant les guerres balkaniques, l'école de Volos fonctionnait comme hôpital qui accueillait des centaines de blessés venus du front. 


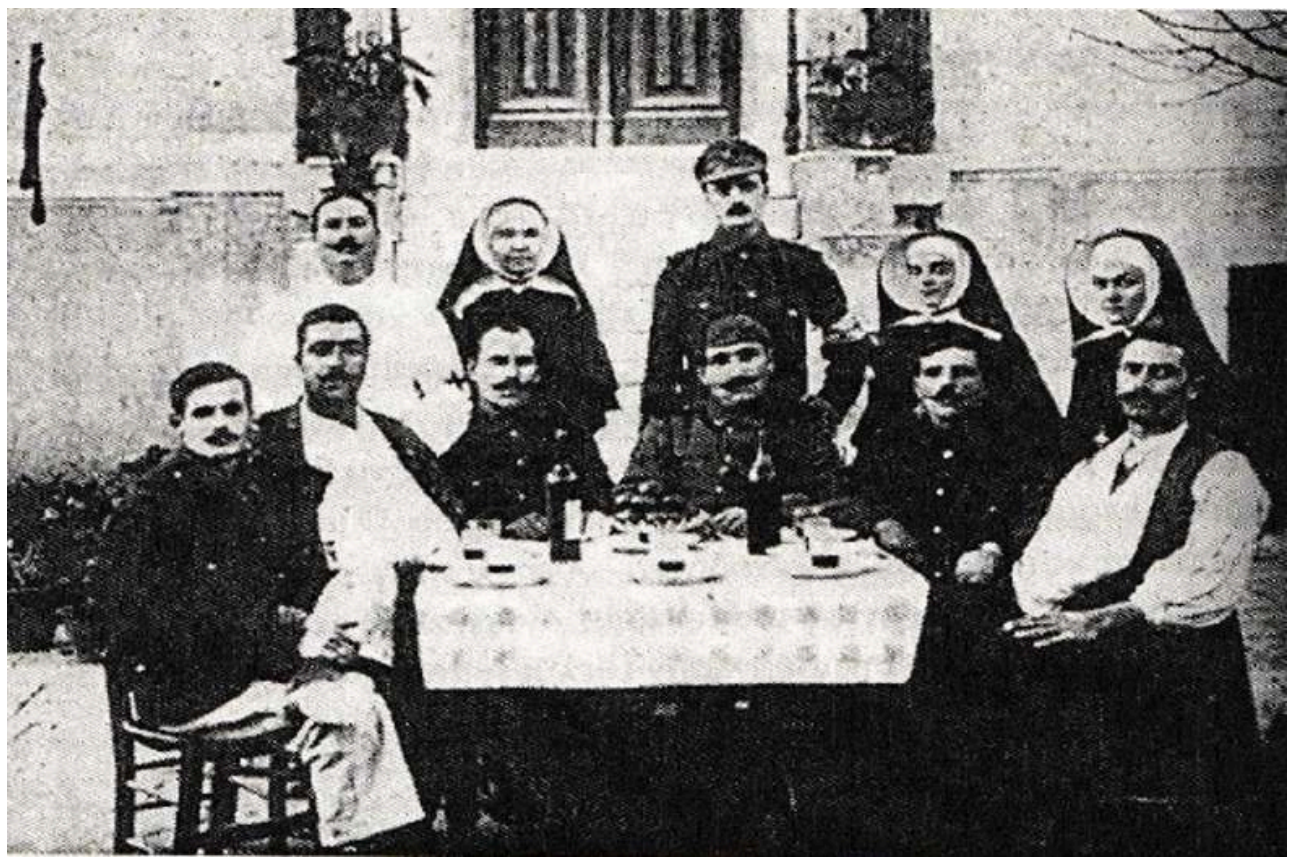

École des Sœurs de Saint Joseph à Volos en 1913 : des soldats blessés pendant les guerres balkaniques dans la cour de l'école (Charitos 2001 : 234).

La même école avait accueilli un grand nombre d'enfants serbes et beaucoup de réfugiés, expatriés de leur pays à cause de la guerre. L'école Jeanne d'Arc du Pirée fonctionnait comme hôpital pendant les guerres balkaniques de 1914-1918, et pendant la Seconde Guerre mondiale. Dans ses installations se trouvait aussi un orphelinat pour enfants pauvres de diverses nationalités et de divers dogmes : orthodoxes, catholiques, israélites.

23 Au début du XXe siècle, le Pirée devient le débouché de l'Asie mineure, lors de la défaite des Grecs en 1922; la catastrophe a eu comme conséquence le départ de milliers de réfugiés grecs qui habitaient en Asie Mineure, qui, faisant escale dans les îles, s'embarquaient par la suite pour le Pirée. Avec eux, des Arméniens et des juifs persécutés y parvenaient aussi. Cet afflux transforma la structure démographique, économique et sociale de la Grèce et, dans notre cas, de la région du Pirée où s'installèrent subitement cent mille réfugiés, dénués de tout. Le rôle de l'école Saint Joseph de l'Apparition fut crucial quant à l'intégration des enfants réfugiés dans la nouvelle patrie ${ }^{15}$. Selon les Historiques, un grand nombre de filles de divers dogmes et nationalités y fut scolarisé à titre gratuit :

En septembre, la défaite des Grecs en Asie Mineure nous amène une foule de réfugiés auxquels nous donnons l'hospitalité pendant plusieurs jours. Nous recevons un bon nombre d'orphelines que nous hébergeons. Un peu plus tard, nous placerons les grandes dans de bonnes familles et les petites nous les garderons pour les élever. La Providence nous viendra en aide. (Historiques I : 186)

Pendant la Seconde Guerre mondiale, le Pirée a été impitoyablement bombardé, Athènes étant épargnée grâce aux antiquités classiques qu'elle abritait. Lors des bombardements du Pirée, deux sœurs ont été tuées et une partie de l'école fut détruite ${ }^{16}$. Sœur Blandine, qui a aujourd'hui 88 ans, se souvient des jours de l'occupation allemande au Pirée: "Nous offrions tous les jours des soupes populaires dans la cour de l'école. Les gens y arrivaient par centaines... Quand on avait les moyens, 
on faisait toujours des soupes... L'action philanthropique s'exerçait sur les enfants et les orphelins, surtout les orphelins $»^{17}$.

\section{Conclusion}

L'objectif principal de la congrégation de Saint Joseph de l'Apparition en Grèce était l'éducation donnée dans un cadre philanthropique. Cette combinaison de philanthropie, d'assistance sociale et d'enseignement, était l'une des raisons principales de l'intégration et de la consécration des Sœurs de Saint Joseph au sein de la société grecque. Æuvrer assidûment dans les domaines de l'éducation et des activités caritatives, c'est là que résident les raisons principales de l'intégration des Sœurs de Saint Joseph au sein de la société du Pirée et la reconnaissance de cette société envers les sœurs de la congrégation.

Pour les sœurs de la congrégation au Pirée, les difficultés furent nombreuses en raison des guerres et des conflits continuels qui ont affligé le pays tout au long du $\mathrm{XX}^{\mathrm{e}}$ siècle ; les besoins financiers pour pouvoir continuer leur œuvre pour les pauvres et les difficultés rencontrées dans leur relation avec l'église orthodoxe, souvent méfiante envers leur œuvre éducative, furent deux difficultés supplémentaires que les sœurs durent affronter. Procurant une éducation de haut niveau, la communauté des sœurs de l'école Jeanne d'Arc a étendu discrètement son apostolat quelle que soit l'appartenance ethnique et religieuse de ses élèves en tissant des réseaux qui durent encore de nos jours.

L'école des Sœurs de Saint Joseph de l'Apparition du Pirée constituait une micrographie de la ville du Pirée, puisqu'il s'agissait d'une école où étaient représentées plusieurs classes sociales, plusieurs croyances religieuses et plusieurs ethnicités; la présence étrangère était très faible jusqu'à la fin du XIX ${ }^{\mathrm{e}}$ siècle, à l'image de la ville du Pirée. Les élèves qui étaient scolarisées venaient du Pirée et de ses alentours, mais aussi de tout le pays, les îles des Cyclades ayant une grande représentation, comme aussi des communautés grecques de l'empire ottoman, Constantinople et Smyrne. La diversité des dogmes adoptés par les élèves, avec le dogme chrétien d'Orient au premier rang, démontre combien l'école était reconnue et estimée tant par les familles grecques orthodoxes du Pirée et d'autres régions, que par des familles d'autres dogmes et croyances (Lianou 2013: 536). La diversité ethnique que l'école présentait surtout au début mais aussi tout au long du $\mathrm{XX}^{\mathrm{e}}$ siècle, consacre sa présence comme un lieu éducatif reconnu largement et comme un facteur d'influence sur la société et les composants éducatifs de cette époque.

Depuis la moitié du XIX ${ }^{e}$ siècle, cette école représente le seul facteur de la civilisation française et européenne au Pirée; c'est la raison principale pour laquelle la classe moyenne bourgeoise envoyait ses filles dans cette école, pour qu'elles se familiarisent avec la civilisation française et apprennent la langue française. L'enseignement du français reste central dans le curriculum de l'école depuis sa création. La protection de l'ambassade de France et la collaboration avec l'École française d'Athènes, la plus ancienne institution scientifique en Grèce, étaient une sorte de reconnaissance du travail effectué au sein de l'école : un niveau élevé dans l'enseignement offert et un très bon niveau d'apprentissage de la langue française. 

l'Apparition est notoire, la reconnaissance de leur œuvre qui a été couronnée par le prix de l'Académie hellénique en témoigne. L'Académie hellénique a en effet honoré en 2009, l'école Jeanne d'Arc pour sa présence éducative et philanthropique au Pirée durant cent cinquante ans, de 1859 à nos jours ${ }^{18}$, et lui a rendu hommage pour avoir éduqué la jeunesse du Pirée, favorisé une éducation ouverte à la différence et assisté les habitants de la ville pendant les périodes difficiles de l'histoire néo-hellénique ${ }^{19}$.

\section{BIBLIOGRAPHIE}

ANTONIOU, David (2011). Écoles françaises en Grèce. Essai d'inventaire. Athènes : Centre International de Recherche Ésope-La Fontaine.

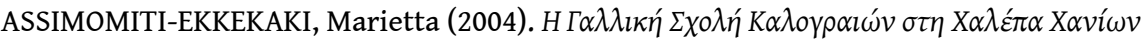
(1852-1983) [L'école française des sœurs à Halépa, La Canée (1852-1983)]. Réthymnon.

CABANEL, Patrick (2006). «Trois France en Méditerranée orientale : l'empire immatériel de la langue ». In Patrick Cabanel (dir.), Une France en Méditerranée, écoles, langues et cultures françaises $X I X^{e}, X^{e}$ siècles. Paris : Créaphis, 9-29.

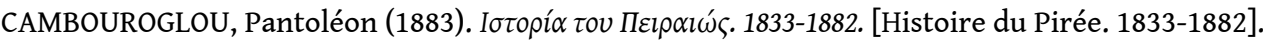
Athènes : Typografeio $O$ Asmodaios.

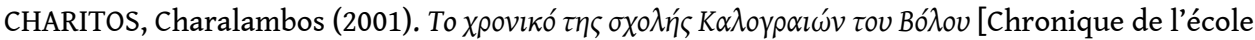
des Sœurs à Volos]. Athènes : Ellinika Grammata.

DUFOURCQ, Elisabeth. (2009). Les aventurières de Dieu. Trois siècles d'histoire missionnaire française, Paris : Perrin/collection Tempus.

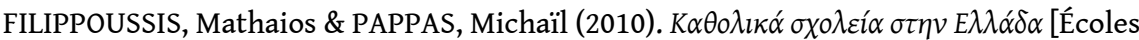
catholiques en Grèce]. Thessaloniki : Ekdoseis Apostolikou Vikariatou/Analecta Historica-3.

FLITOURIS, Lampros (2005). «La présence culturelle française en Grèce dans les années 1940» [H

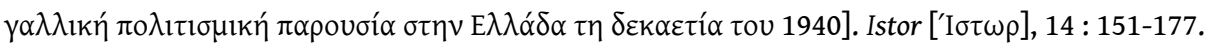

KAVALLIERAKIS, Stéphanos (2008). Éducation et écoles étrangères en Grèce au XIX ${ }^{e}$ siècle. Le cas des écoles de Sours de Saint Joseph de l'Apparition (1856-1893). Thèse de doctorat présentée à l'Université Marc Bloch, France (thèse non publiée).

LACROIX, M. L'abbé (1898). L'école française des filles au Pirée. Brest : Imprimerie de la Presse Catholique du Finistère.

LANGLOIS, Claude (1984). Le catholicisme au féminin. Les congrégations françaises à supérieure générale au XIX ${ }^{e}$ siècle. Paris : CERF.

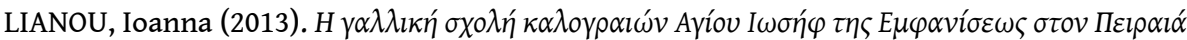
(1859-1940) [L'École française des Sœurs Saint Joseph de l'Apparition au Pirée]. Thèse de doctorat présentée à l'Université Panteion, Athènes (thèse non publiée). 
MOLHO, Rena (2003). « Les Juifs en Grèce au XX $\mathrm{X}^{\mathrm{e}}$ siècle ». Matériaux pour l'histoire de notre temps, $71: 39-48$.

MYROGIANNIS, Stratos (2012). The Emergence of a Greek Identity (1700-1821). London : Cambridge Scholars Publishing.

PROVATA, Despina (2011). « Construction identitaire et enseignement du français en Grèce au $\mathrm{XIX}^{\mathrm{e}}$ siècle ». In Konstantinos Dimadis (dir.) Identities in the Greek World (from 1204 to the present day), Actes du $4^{e}$ Congrès européen d'études néo-helléniques (Grenade, 9-11/9/2010), vol. 5. Athènes : European Society of Modern Greek Studies, 281-192.

PROVATA, Despina (2012). « Enseignement féminin et apprentissage du français en Grèce au XIX ${ }^{\mathrm{e}}$ siècle ». Documents pour l'histoire du français langue étrangère ou seconde, 47-48, 174-187.

SANCHEZ-SUMMERER, Karène (2010). « Les catholiques palestiniens et la langue française (1870-1950) ». Documents pour l'histoire du français langue étrangère ou seconde, 45 : 17-39.

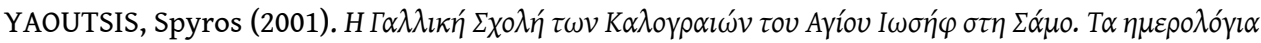
$\tau \eta \varsigma \pi \rho \circ \sigma \varphi v \gamma l \alpha ́ \varsigma \kappa \alpha \alpha l \tau o v \pi 0 \lambda \varepsilon ́ \mu o v$ [L'école française des Sœurs de Saint Joseph à Samos]. Athènes : Arcudius.

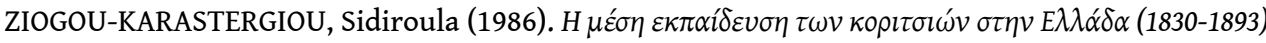
[L'enseignement secondaire des filles en Grèce (1830-1893)]. Athènes : IAEN.

\section{NOTES}

1. On peut consulter, à titre d'exemple, Patrick Cabanel (2006:9-29); Karène Sanchez-Summerer (2010: 17-39).

2. «It is exactly this pragmatic aspect of the Enlightenment Project and the social implementation of Enlightenment doctrines that makes the Greek case an interesting field of investigation since Greek-speaking intellectuals transformed Enlightenment doctrines into a practical nationalist ideology to claim their national independence. The Greek case exemplifies the weak points of the naïve Enlightenment cosmopolitanism » (Myrogiannis 2012 : 20).

3. Par exemple, le catéchisme sur le dogme orthodoxe par un professeur spécialisé ou par un prêtre orthodoxe, est introduit comme matière obligatoire dans les écoles grecques en 1836 ; cet acte fera l'objet d'un débat continuel entre les écoles étrangères, protestantes et catholiques, et l'État hellénique, pendant plus d'un siècle - ces écoles s'opposant souvent à des directives essentielles du système éducatif central.

4. Les tensions politiques entre l'état hellénique et les pays européens avaient une influence négative sur les relations des écoles catholiques avec les autorités politiques. Une tension dans les relations franco-helléniques a même conduit à la suspension des activités éducatives de l'école des sœurs de Saint Joseph de l'Apparition à Athènes, de 1859 à 1863, à cause des accusations de prosélytisme lancées contre la congrégation (Kavallierakis 2008 : 193-194).

5. L'ordonnance 284/1968, la loi 682/1977, la loi 1566/1985, et la Décision du 12/5/1999: la ratification du programme culturel entre la Grèce et la France, ont conditionné le fonctionnement des écoles congréganistes en Grèce.

6. En 1883, la population catholique dans les îles des Cyclades comptait presque 14000 personnes; dans les îles de Tinos, Syros et Santorin, il y avait des sièges d'évêques catholiques tandis que Naxos était le siège de l'archevêché catholique (Filippoussis \& Pappas 2010:48). Aujourd'hui le nombre de Grecs catholiques remonte à 50000 personnes, dont plus de la moitié habitent dans les îles de la mer Egée. <http://www.cathecclesia.gr/hellas/index.php/ arxiepiskopi-naksou-tinou> (22 mai 2015). 
7. C'est le point de vue de la plupart des chercheurs qui ont étudié la présence des écoles des sœurs catholiques en Grèce. Il faut mentionner que les écoles catholiques des sœurs en Grèce enseignement primaire et secondaire - n'ont pas fait l'objet d'études exhaustives. En ce qui concerne la congrégation de Saint Joseph de l'Apparition, il y a deux livres sur l'histoire des écoles dans l'île de Samos et à La Canée en Crète. La thèse non publiée de S. Kavallierakis (2008) étudie les écoles de la congrégation de Saint Joseph de l'Apparition jusqu'en 1893. L'école du Pirée quant à elle fait l'objet de la thèse de doctorat non publiée de I. Lianou (2013) qui se réfère à la structure pédagogique, aux curricula, à l'enseignement du français dans l'école, et à d'autres thèmes qui intéressent la vie scolaire et la structure de l'enseignement.

8. Les Historiques I portent sur la période de 1859 à 1930 et Historiques II sur celle de 1931 à 1975.

9. L'Alliance israélite universelle avait fondé des écoles juives dans les villes grecques où il y avait une forte présence juive : la première fut fondée à Volos, en 1864, puis à Salonique, à Ioannina et à Larissa. À Salonique, où la population juive était nombreuse et active, l'école fut fondée en 1873. Il y avait dans cette ville, à la fin de la Première Guerre mondiale « douze écoles juives de qualité, dont quatre établissement secondaires" (Molho 2003: 40). Pendant l'entre-deux-guerres et surtout après la Seconde Guerre mondiale, toutes les écoles de l'Alliance ont cessé d'exister l'une après l'autre et en 1960 aucune ne fonctionnait plus.

10. Sur l'expansion des communautés religieuses féminines dans le monde entier afin d'essaimer et d'assister les populations locales, voir Dufourcq 2009.

11. La loi de 1834, sur les écoles primaires, prévoyait aussi la scolarisation des filles alors que celle de 1929 autorisait leur accès à l'enseignement secondaire.

12. Décrets du 31 décembre 1836 et du 12 janvier 1837 sur l'organisation des Écoles helléniques et des Gymnases.

13. Il faut mentionner qu'il existait aussi des écoles de filles fondées par des particuliers, comme l'école du couple protestant américain Hill à Athènes ou l'école du protestant allemand Hildner à Syros (Syra).

14. Il s'agissait des trois actions à accomplir pour les sœurs de la congrégation Saint Joseph de l'Apparition, comme il est écrit dans une lettre de la sœur Blandine Dréant envoyée à sa famille en France ; la sœur Blandine me l'a confiée, durant une interview au Pirée, à l'école Jeanne d'Arc, le 14 novembre 2014. La sœur Blandine se trouve aujourd'hui à Volos; elle est arrivée en Grèce en 1947 à l'âge de 27 ans, et elle est restée au Pirée jusqu'en 1972. Je la remercie vivement de notre discussion qui m'a beaucoup appris sur la longue histoire de l'école Jeanne d'Arc et sur la vie communautaire des sœurs.

15. Les supérieures reviennent souvent à la question de la défaite en Asie Mineure et aux réfugiés Grecs : « [...] pendant ce même mois [en janvier] les épidémies de typhus exanthématique et de petite vérole prennent des proportions alarmantes, causées par l'agglomération des réfugiés » (Historiques I : 188).

16. M. Asimomiti-Ekkekaki parle des malheurs causés par la guerre et l'occupation allemande à Halépa, en Crète, où se trouvait l'école des sœurs (2004 : 114-121). S. Yaoutsis raconte la réception des réfugiés par l'école des sœurs à Samos et les malheurs causés par la guerre qui avait affecté l'école et ses communautés (2010).

17. Interview avec la sœur Blandine Dréan, le 14 novembre 2014.

18. De nos jours, l'école Jeanne d'Arc au Pirée fonctionne comme école primaire et secondaire privée. L'école française Saint Joseph de Volos fonctionne de 1904 à nos jours comme école primaire privée.

19. Le secrétaire général de l'Académie, le professeur Nikolaos Matsaniotis a souligné dans son discours: "Dans l'histoire de l'école, il faut absolument mentionner tant ce qu'elle a offert pendant les deux guerres mondiales que ses activités caritatives durant sa longue histoire. Aujourd'hui encore, l'école exerce des activités pédagogiques et est active dans les domaines 
sociaux et philanthropiques. L'enseignement de la langue et de la civilisation françaises constitue un domaine où a excellé ses compétences, en obtenant des Prix internationaux ».

\section{RÉSUMÉS}

L'école Jeanne d'Arc, fondée au Pirée en 1859 par les Sœurs de Saint Joseph de l'Apparition est une institution qui fonctionne jusqu'à nos jours en Grèce. Nous examinons la contribution de l'école à l'éducation des filles en Grèce au XIX ${ }^{\mathrm{e}}$ siècle, question éducative et culturelle qui a provoqué beaucoup de débats dans le pays. L'apport des sœurs de la Congrégation à la société du Pirée pendant les périodes de guerre qui ont affligé le pays durant tout le $\mathrm{XX}^{\mathrm{e}}$ siècle est aussi particulièrement important et se fait au nom de leur mission qui comprend des activités de charité et d'éducation. Pour les sœurs de la congrégation Saint Joseph de l'Apparition, il y a trois actions à accomplir : former, soigner, assister.

This article presents the school Jeanne d'Arc, which was established in Piraeus, in 1859 by the French nuns of Saint Joseph de l'Apparition, an institution still in function in Greece. It examines this school's contribution to the girls' education in Greece, in the $19^{\text {th }}$ century, an educational and cultural issue that caused a lot of debate in the country. It also examines the cultural and philanthropic impact of the school on the Piraeus society during wartime which plagued the country in the $20^{\text {th }}$ century. For the nuns of Saint Joseph de l'Apparition, there are three actions to accomplish : educate, provide care, assist.

\section{INDEX}

Keywords : National identity, girls' education, cultural impact, confessional schools, Orthodox Church

Mots-clés : Identité nationale, éducation des filles, impact culturel, écoles congréganistes, Église orthodoxe

\section{AUTEURS}

\section{VASSILIKI LALAGIANNI}

Université du Péloponnèse 